\title{
New Information Inequalities in Terms of One Parametric Generalized Divergence Measure and Application
}

\author{
K. C. Jain ${ }^{1, *}$, Praphull Chhabra ${ }^{1,+}$ \\ ${ }^{1}$ Department of Mathematics, Malaviya National Institute of Technology, Jaipur (Rajasthan), India \\ jainkc_2003@yahoo.com \\ +prfl24@gmail.com,pcnitj@yahoo.com
}

Article history:

Received December 2014

Accepted January 2015

Available online January 2015

\begin{abstract}
In this work, firstly we introduce the new information divergence measure, characterize it and get the mathematical relations with other divergences. Further, we introduce new information inequalities on the new generalized f- divergence measure in terms of the well-known one parametric generalized divergence. Further, we obtain bounds of the new divergence and the Relative J- divergence as an application of new information inequalities by using Logarithmic power mean and Identric mean, together with numerical verification by taking two discrete probability distributions: Binomial and Poisson. Approximate relations of the new divergence and Relative J- divergence with Chi- square divergence, have been obtained respectively.
\end{abstract}

Index terms: New divergence; New information inequalities; Parametric generalized divergence; Bounds; Logarithmic power mean; Identric mean; Binomial and Poisson distributions; Asymptotic approximation.

Mathematics Subject Classification: Primary 94A17, Secondary 26D15.

\section{Introduction}

Divergence measures are basically measures of distance between two probability distributions or compare two probability distributions, i.e., divergence measures are directly propositional to the distance between two probability distributions. It means that any divergence measure must take its minimum value zero when probability distributions are equal and maximum when probability distributions are perpendicular to each other. So, any divergence measure must increase as probability distributions move apart. Depending on the nature of the problem, different divergence measures are suitable. So it is always desirable to develop a new divergence measure. 
Divergence measures have been demonstrated very useful in a variety of disciplines such as economics and political science [34, 35], biology [26], analysis of contingency tables [14], approximation of probability distributions $[\mathbf{8}, \mathbf{2 3}]$, signal processing $[\mathbf{2 1}, \mathbf{2 2}]$, pattern recognition $[\mathbf{2}, \mathbf{7}, \mathbf{2 0}]$, color image segmentation [24], 3D image segmentation and word alignment [33], cost- sensitive classification for medical diagnosis [28], magnetic resonance image analysis [36] etc.

Also we can use divergences in fuzzy mathematics as fuzzy directed divergences and fuzzy entropies [1, $15,19]$, which are very useful to find the amount of average ambiguity or difficulty in making a decision whether an element belongs to a set or not. Fuzzy information measures have recently found applications to fuzzy aircraft control, fuzzy traffic control, engineering, medicines, computer science, management and decision making etc.

Without essential loss of insight, we have restricted ourselves to discrete probability distributions, so let $\Gamma_{n}=\left\{P=\left(p_{1}, p_{2}, p_{3} \ldots, p_{n}\right): p_{i}>0, \sum_{i=1}^{n} p_{i}=1\right\}, n \geq 2$ be the set of all complete finite discrete probability distributions. The restriction here to discrete distributions is only for convenience, similar results hold for continuous distributions. If we take $p_{i} \geq 0$ for some $i=1,2,3, \ldots, n$, then we have to suppose that $0 f(0)=0 f\left(\frac{0}{0}\right)=0$.

Some generalized functional information divergence measures had been introduced, characterized and applied in variety of fields, such as: Csiszar's $f$ - divergence [9, 10], Bregman's $f$ - divergence [5], Burbea- Rao's $f$-divergence [6], Renyi's like $f$ - divergence [27] etc. Similarly, Jain and Saraswat [18] defined new generalized $f$-divergence measure, which is given by

$$
S_{f}(P, Q)=\sum_{i=1}^{n} q_{i} f\left(\frac{p_{i}+q_{i}}{2 q_{i}}\right)
$$

where $f:(0, \infty) \rightarrow R$ (set of real no.) is real, continuous, and convex function and $P=\left(p_{1}, p_{2}, p_{3} \ldots, p_{n}\right), Q=\left(q_{1}, q_{2}, q_{3} \ldots, q_{n}\right) \in \Gamma_{n}$, where $p_{i}$ and $q_{i}$ are probability mass functions. The advantage of these generalized divergences is that many divergence measures can be obtained from these generalized $f$ - measures by suitably defining the function $f$. Some resultant divergences by $S_{f}(P, Q)$, are as follows.

a. If we take $f(t)=(t-1) \log t$ in (1.1), we obtain

$$
S_{f}(P, Q)=\frac{1}{2} \sum_{i=1}^{n}\left(p_{i}-q_{i}\right) \log \left(\frac{p_{i}+q_{i}}{2 q_{i}}\right)=\frac{1}{2} J_{R}(P, Q)
$$

where $J_{R}(P, Q)$ is called the Relative $\mathrm{J}$ - divergence [12].

b. If we take $f(t)=[s(s-1)]^{-1}\left(t^{s}-1\right)=\phi_{s}(t), s \in R-\{0,1\}$ in (1.1), we obtain 


$$
\begin{aligned}
& \Phi_{s}(Q, P)=[s(s-1)]^{-1}\left[\sum_{i=1}^{n} q_{i}\left(\frac{p_{i}+q_{i}}{2 q_{i}}\right)^{s}-1\right] \\
& = \begin{cases}\frac{1}{4} \sum_{i=1}^{n} \frac{\left(p_{i}-q_{i}\right)^{2}}{p_{i}+q_{i}}=\frac{1}{4} \Delta(P, Q)=\Phi_{-1}(Q, P), & s=-1 \\
\sum_{i=1}^{n} q_{i} \log \left(\frac{2 q_{i}}{p_{i}+q_{i}}\right)=F(Q, P)=\lim _{s \rightarrow 0} \Phi_{s}(Q, P), & s=0 \\
\sum_{i=1}^{n}\left(\frac{p_{i}+q_{i}}{2}\right) \log \left(\frac{p_{i}+q_{i}}{2 q_{i}}\right)=G(Q, P)=\lim _{s \rightarrow 1} \Phi_{s}(Q, P), s=1 \\
\frac{1}{8} \sum_{i=1}^{n} \frac{\left(p_{i}-q_{i}\right)^{2}}{q_{i}}=\frac{1}{8} \chi^{2}(P, Q)=\Phi_{2}(Q, P), & s=2\end{cases}
\end{aligned}
$$

where $\Phi_{s}(Q, P), s \in R-\{0,1\}$ is one parametric generalized divergence measure, which is known as adjoint of the Unified Relative Jensen- Shannon and Arithmetic- Geometric divergence measure of type " $s^{\prime} \Phi_{s}(P, Q)$ [32], $s$ is called parameter and $\Delta(P, Q)$ is the Triangular discrimination [11], $F(Q, P)$ is adjoint of the Relative Jensen- Shannon divergence $F(P, Q)$ [29], $G(Q, P)$ is adjoint of the Relative Arithmetic- Geometric divergence $G(P, Q)$ [31], and $\chi^{2}(P, Q)$ is the Chi- Square divergence (Pearson div. measure) [25].

Similarly, we can obtain many divergences by using linear convex functions. Since these divergences are not worthful in practice, therefore we can skip them.

Now, there are two generalized means which are being used in this paper for calculations only. This are as follows.

$$
\begin{aligned}
& L_{p}(a, b)=\left\{\begin{array}{ll}
\frac{b^{p+1}-a^{p+1}}{(p+1)(b-a)}, & p \neq-1,0 \\
\frac{\log b-\log a}{b-a}, & p=-1
\end{array} \quad a, b>0, a \neq b .\right. \\
& I, \quad a, b)= \begin{cases}\left\{\frac{1}{e}\left(\frac{b^{b}}{a^{a}}\right)^{\frac{1}{b-a}},\right. & a \neq b \quad a, b>0 . \\
b, & a=b\end{cases}
\end{aligned}
$$

Means (1.4) and (1.5) are called $p$ - Logarithmic power mean and Identric mean respectively. 


\section{New information divergence measure, properties and relations}

In this section, we introduce new information divergence measure of class $S_{f}(P, Q)$. Properties and relations of this new divergence with other divergences are also given. Let us start with definition of convex function.

Convex function: A function $f(x)$ is said to be convex over an interval $(a, b)$ if for every $x_{1}, x_{2} \in(a, b)$ and $0 \leq \lambda \leq 1$, we have $f\left[\lambda x_{1}+(1-\lambda) x_{2}\right] \leq \lambda f\left(x_{1}\right)+(1-\lambda) f\left(x_{2}\right)$, and said to be strictly convex if equality does not hold only if $\lambda \neq 0$ or $\lambda \neq 1$.

The following properties (Theorems 2.1,2.2, and 2.3) of new generalized $f$-divergence $S_{f}(P, Q)$ and their proofs can be seen in literature [18], so we omit the details.

Theorem 2.1 (Positivity) Let $f: R_{+} \rightarrow R$ be a real, convex function and $(P, Q) \in \Gamma_{n} \times \Gamma_{n}$, then we have

$S_{f}(P, Q) \geq f(1)$.

If $f$ is normalized, i.e., $f(1)=0$ then $S_{f}(P, Q) \geq 0$, and if $f$ is strictly convex, equality holds in (2.1) if and only if $p_{i}=q_{i} \forall i=1,2,3, \ldots n$, i.e., $S_{f}(P, Q) \geq 0$ and $S_{f}(P, Q)=0$ if and only if $P=Q$.

Theorem 2.2 (Linearity property) If $f_{1}$ and $f_{2}$ are two convex functions such that $F=a f_{1}+b f_{2}$ then $S_{F}(P, Q)=a S_{f_{1}}(P, Q)+b S_{f_{2}}(P, Q)$, where $a$ and $b$ are constants and $(P, Q) \in \Gamma_{n} \times \Gamma_{n}$.

Theorem 2.3 (Relation with Csiszar's $f$ - divergence) Let $f: R_{+} \rightarrow R$ be a convex and normalized function, i.e., $f^{\prime \prime}(t) \geq 0 \forall t>0$ and $f(1)=0$ respectively then for $(P, Q) \in \Gamma_{n} \times \Gamma_{n}$, we have

$S_{f}(P, Q) \leq C_{f}(P, Q) \leq E_{C_{f}}(P, Q)$,

where $S_{f}(P, Q)$ is given by $(1.1), C_{f}(P, Q)$ is well known Csiszar's $f$ - divergence, given by

$$
C_{f}(P, Q)=\sum_{i=1}^{n} q_{i} f\left(\frac{p_{i}}{q_{i}}\right),
$$

and

$$
E_{C_{f}}(P, Q)=\sum_{i=1}^{n}\left(p_{i}-q_{i}\right) f^{\prime}\left(\frac{p_{i}}{q_{i}}\right) .
$$

Now, let $f: R_{+} \rightarrow R$ be a mapping defined as

$$
f(t)=\frac{t+1}{2} \log \left(\frac{t+1}{2 t}\right),
$$




$$
\begin{aligned}
& f^{\prime}(t)=\frac{1}{2}\left[\log \left(\frac{t+1}{2 t}\right)-\frac{1}{t}\right], f(1)=0 \text { and } \\
& f^{\prime \prime}(t)=\frac{1}{2 t^{2}(t+1)}>0 \forall t \in R_{+} .
\end{aligned}
$$

Since $f^{\prime \prime}(t)>0 \forall t>0$ and $f(1)=0$, so $f(t)$ is convex and normalized function respectively. Now put $f(t)$ in (1.1), we get

$S_{f}(P, Q)=\sum_{i=1}^{n}\left(\frac{p_{i}+3 q_{i}}{4}\right) \log \left[\frac{p_{i}+3 q_{i}}{2\left(p_{i}+q_{i}\right)}\right]=M^{*}(P, Q)$.

We conclude the followings for new divergence $M^{*}(P, Q)$.

a. In view of theorem 2.1 , we can say that $M^{*}(P, Q)>0$ and is convex in the pair of probability distribution $(P, Q) \in \Gamma_{n} \times \Gamma_{n}$.

b. $M^{*}(P, Q)=0$ if and only if $P=Q$ or $p_{i}=q_{i}$ (attending its minimum value).

c. $M^{*}(P, Q) \neq M^{*}(Q, P) \Rightarrow M^{*}(P, Q)$ is non- symmetric divergence measure.

Now, we derive two simple relations (Propositions 2.1 and 2.2) for $M^{*}(P, Q)$.

Proposition 2.1 Let $(P, Q) \in \Gamma_{n} \times \Gamma_{n}$, then we have the following new inter relation

$$
M^{*}(P, Q) \geq \frac{1}{4}[F(Q, P)-G(Q, P)]
$$

where $M^{*}(P, Q)$ is given by (2.7) and $F(Q, P), G(Q, P)$ together are given by (1.3).

Proof: Since we know that $A M \geq G M$, i.e., for $a, b>0$

$\frac{a+b}{2} \geq \sqrt{a b}$

Now put $a=1$ and $b=\frac{1}{t}$ in (2.9) for $t>0$, we get

$$
\frac{1+\frac{1}{t}}{2} \geq \sqrt{\frac{1}{t}} \Rightarrow \frac{t+1}{2 t} \geq\left(\frac{1}{t}\right)^{1 / 2} \Rightarrow \log \left(\frac{t+1}{2 t}\right) \geq \frac{1}{2} \log \frac{1}{t} .
$$

Now multiply (2.10) by $\frac{t+1}{2}$ for $t>0$, we get 
$\frac{t+1}{2} \log \left(\frac{t+1}{2 t}\right) \geq \frac{t+1}{4} \log \frac{1}{t}$

Now put $t=\frac{p_{i}+q_{i}}{2 q_{i}}, i=1,2,3, \ldots, n$ in (2.11), multiply by $q_{i}$ and then sum over all $i=1,2,3, \ldots, n$, we get $\sum_{i=1}^{n}\left(\frac{p_{i}+3 q_{i}}{4}\right) \log \left[\frac{p_{i}+3 q_{i}}{2\left(p_{i}+q_{i}\right)}\right] \geq \sum_{i=1}^{n}\left(\frac{p_{i}+3 q_{i}}{8}\right) \log \left[\frac{2 q_{i}}{\left(p_{i}+q_{i}\right)}\right]$, i.e.,

$M^{*}(P, Q) \geq \sum_{i=1}^{n} \frac{1}{4}\left(\frac{p_{i}+q_{i}+2 q_{i}}{2}\right) \log \left[\frac{2 q_{i}}{\left(p_{i}+q_{i}\right)}\right]$, i.e.,

$M^{*}(P, Q) \geq \frac{1}{4}\left[\sum_{i=1}^{n} q_{i} \log \frac{2 q_{i}}{p_{i}+q_{i}}-\sum_{i=1}^{n} \frac{p_{i}+q_{i}}{2} \log \frac{p_{i}+q_{i}}{2 q_{i}}\right]$, i.e.,

$M^{*}(P, Q) \geq \frac{1}{4}[F(Q, P)-G(Q, P)]$. Hence prove the inequality (2.8).

Proposition 2.2 Let $(P, Q) \in \Gamma_{n} \times \Gamma_{n}$, then we have the following new inter relation.

$M^{*}(P, Q) \leq \frac{1}{2}\left[J_{R}(Q, P)+\chi^{2}(Q, P)\right]$

where $M^{*}(P, Q), J_{R}(Q, P)$ and $\chi^{2}(Q, P)$ are given by (2.7), (1.2), and (1.3) respectively.

Proof: Since we know the following by (2.2).

$S_{f}(P, Q) \leq E_{C_{f}}(P, Q) \Rightarrow \sum_{i=1}^{n} q_{i} f\left(\frac{p_{i}+q_{i}}{2 q_{i}}\right) \leq \sum_{i=1}^{n}\left(p_{i}-q_{i}\right) f^{\prime}\left(\frac{p_{i}}{q_{i}}\right)$.

Now put $f(t)$ and $f^{\prime}(t)$ in (2.13), we get

$$
\begin{aligned}
& \sum_{i=1}^{n} \frac{p_{i}+3 q_{i}}{4} \log \frac{p_{i}+3 q_{i}}{2\left(p_{i}+q_{i}\right)} \leq \frac{1}{2} \sum_{i=1}^{n}\left(p_{i}-q_{i}\right)\left[\log \left(\frac{p_{i}+q_{i}}{2 p_{i}}\right)-\frac{q_{i}}{p_{i}}\right], \text { i.e., } \\
& M^{*}(P, Q) \leq \frac{1}{2}\left[\sum_{i=1}^{n}\left(p_{i}-q_{i}\right) \log \left(\frac{p_{i}+q_{i}}{2 p_{i}}\right)+\sum_{i=1}^{n} \frac{\left(q_{i}-p_{i}\right) q_{i}}{p_{i}}\right], \text { i.e, } \\
& M^{*}(P, Q) \leq \frac{1}{2}\left[J_{R}(Q, P)+\sum_{i=1}^{n} \frac{\left(q_{i}-p_{i}\right) q_{i}}{p_{i}}\right], \text { i.e., }
\end{aligned}
$$




$$
\begin{aligned}
& M^{*}(P, Q) \leq \frac{1}{2}\left[J_{R}(Q, P)+\sum_{i=1}^{n}\left[\frac{\left(q_{i}-p_{i}\right)^{2}}{p_{i}}+\frac{p_{i}\left(q_{i}-p_{i}\right)}{p_{i}}\right]\right], \text { i.e., } \\
& M^{*}(P, Q) \leq \frac{1}{2}\left[J_{R}(Q, P)+\chi^{2}(Q, P)\right],\left(\because \sum_{i=1}^{n} p_{i}=\sum_{i=1}^{n} q_{i}=1\right) .
\end{aligned}
$$

Hence prove the inequality (2.12).

Remark: By taking together (2.8) and (2.12), we get

$$
\frac{1}{4}[F(Q, P)-G(Q, P)] \leq M^{*}(P, Q) \leq \frac{1}{2}\left[J_{R}(Q, P)+\chi^{2}(Q, P)\right] .
$$

\section{New information inequalities}

In this section, we introduce two new information inequalities (Theorems 3.1 and 3.2) on $S_{f}(P, Q)$; one of them is in terms of one parametric divergence $\Phi_{s}(Q, P)$. Such inequalities are for instance needed in order to calculate the relative efficiency of two divergences.

Theorem 3.1 Let $f: R_{+} \rightarrow R$ be a real, convex function on $(\alpha, \beta) \subset R_{+}$with $0<\alpha \leq 1 \leq \beta<\infty, \alpha \neq \beta$.

If $P, Q \in \Gamma_{n}$ and satisfying the assumption $0<\alpha<\frac{1}{2} \leq \frac{p_{i}+q_{i}}{2 q_{i}} \leq \beta<\infty \forall i=1,2,3, \ldots, n$, then we have the following inequalities

$$
S_{f}(P, Q) \leq B_{f}(\alpha, \beta)
$$

where $S_{f}(P, Q)$ is given by $(1.1)$ and

$$
B_{f}(\alpha, \beta)=\frac{(\beta-1) f(\alpha)+(1-\alpha) f(\beta)}{\beta-\alpha} .
$$

Proof: Since $f$ is convex on $(0, \infty)$, therefore we can write the following for $(\alpha, \beta) \in R_{+} \times R_{+}, \lambda \in[0,1]$ by the definition of convex function

$$
f[\lambda \alpha+(1-\lambda) \beta] \leq \lambda f(\alpha)+(1-\lambda) f(\beta) .
$$

Now assume $\lambda=\frac{\beta-x}{\beta-\alpha}$ for $x \in(\alpha, \beta)$ in (3.3), we get

$$
f(x) \leq \frac{(\beta-x) f(\alpha)+(x-\alpha) f(\beta)}{\beta-\alpha} .
$$


Now put $x=\frac{p_{i}+q_{i}}{2 q_{i}}$ in (3.4), multiply by $q_{i}$ and then sum over all $i=1,2, \ldots n$, we obtain the require inequality (3.1).

Theorem 3.2 Let $f: R_{+} \rightarrow R$ be a real, convex and twice differentiable function on $(\alpha, \beta) \subset R_{+}$with $0<\alpha \leq 1 \leq \beta<\infty, \alpha \neq \beta$. If there exists the real constants $m, M$ with $m<M$ and

$$
m \leq t^{2-s} f^{\prime \prime}(t) \leq M \text { for all } t \in(\alpha, \beta), s \in R-\{0,1\} .
$$

If $P, Q \in \Gamma_{n}$ and satisfying the assumption $0<\alpha<\frac{1}{2} \leq \frac{p_{i}+q_{i}}{2 q_{i}} \leq \beta<\infty \forall i=1,2,3, \ldots, n$, then we have

$$
m\left[B_{\phi_{s}}(\alpha, \beta)-\Phi_{s}(Q, P)\right] \leq B_{f}(\alpha, \beta)-S_{f}(P, Q) \leq M\left[B_{\phi_{s}}(\alpha, \beta)-\Phi_{s}(Q, P)\right]
$$

where $S_{f}(P, Q), \Phi_{s}(Q, P)$ are given by (1.1) and (1.3) respectively, and

$$
\begin{aligned}
& B_{f}(\alpha, \beta)=\frac{(\beta-1) f(\alpha)+(1-\alpha) f(\beta)}{\beta-\alpha} . \\
& B_{\phi_{s}}(\alpha, \beta)=\frac{(\beta-1) \phi_{s}(\alpha)+(1-\alpha) \phi_{s}(\beta)}{\beta-\alpha} .
\end{aligned}
$$

Proof: Let us define a function $F_{m}: R_{+} \rightarrow R$ as

$$
F_{m}(t)=f(t)-m[s(s-1)]^{-1}\left(t^{s}-1\right)=f(t)-m \phi_{s}(t)
$$

where $\phi_{s}(t)=[s(s-1)]^{-1}\left(t^{s}-1\right), s \in R-\{0,1\}$.

Since $f(t)$ and $\phi_{s}(t)$ are both twice differentiable, therefore $F_{m}(t)$ is twice differentiable as well, So

$F_{m}^{\prime \prime}(t)=f^{\prime \prime}(t)-m t^{s-2}=t^{s-2}\left(t^{2-s} f^{\prime \prime}(t)-m\right) \geq 0$.

Since $F_{m}^{\prime \prime}(t) \geq 0 \forall t \in(\alpha, \beta) \subset(0, \infty)$, therefore $F_{m}(t)$ is convex as well.

Now we write inequality (3.1) for the function $F_{m}(t)$, we obtain

$$
\begin{aligned}
& S_{F_{m}}(P, Q) \leq B_{F_{m}}(\alpha, \beta), \text { i.e., } \\
& \sum_{i=1}^{n} q_{i} f\left(\frac{p_{i}+q_{i}}{2 q_{i}}\right)-m[s(s-1)]^{-1} \sum_{i=1}^{n} q_{i}\left(\frac{p_{i}+q_{i}}{2 q_{i}}\right)^{s}-1
\end{aligned}
$$


$\leq \frac{(\beta-1) f(\alpha)+(1-\alpha) f(\beta)}{(\beta-\alpha)}-m\{s(s-1)\}^{-1} \frac{(\beta-1)\left(\alpha^{s}-1\right)+(1-\alpha)\left(\beta^{s}-1\right)}{(\beta-\alpha)}$, i.e.,

$S_{f}(P, Q)-m \Phi_{s}(Q, P) \leq B_{f}(\alpha, \beta)-m B_{\phi_{s}}(\alpha, \beta)$, i.e.,

$m\left[B_{\phi_{s}}(\alpha, \beta)-\Phi_{s}(Q, P)\right] \leq B_{f}(\alpha, \beta)-S_{f}(P, Q)$.

Hence prove the first inequality of (3.5).

The second inequality of (3.5) obtains by a similar approach for the function

$F_{M}(t)=M[s(s-1)]^{-1}\left(t^{s}-1\right)-f(t)$. We omit the details.

\section{Bounds of the new information divergence measure}

In this section, we obtain bounds of the new information divergence measure $M^{*}(P, Q)$ in terms of one parametric generalized divergence measure $\Phi_{s}(Q, P)$ for different values of $s$, by using new inequalities (3.5) and means (1.4) and (1.5).

Proposition 4 Let $\Phi_{s}(Q, P)$ and $M^{*}(P, Q)$ be defined as in (1.3) and (2.7) respectively. For $P, Q \in \Gamma_{n}$, we have

a. For $s \rightarrow 0,1$ and $s>0$, we have

$\frac{1}{2 \beta^{s}(\beta+1)}\left[B_{\phi_{s}}(\alpha, \beta)-\Phi_{s}(Q, P)\right] \leq B_{f}(\alpha, \beta)-M^{*}(P, Q)$

$\leq \frac{1}{2 \alpha^{s}(\alpha+1)}\left[B_{\phi_{s}}(\alpha, \beta)-\Phi_{s}(Q, P)\right]$.

b. For $s \leq-1$, we have

$\frac{1}{2 \alpha^{s}(\alpha+1)}\left[B_{\phi_{s}}(\alpha, \beta)-\Phi_{s}(Q, P)\right] \leq B_{f}(\alpha, \beta)-M^{*}(P, Q)$

$\leq \frac{1}{2 \beta^{s}(\beta+1)}\left[B_{\phi_{s}}(\alpha, \beta)-\Phi_{s}(Q, P)\right]$,

where $B_{f}(\alpha, \beta)$ and $B_{\phi_{s}}(\alpha, \beta)$ are evaluated below by equations (4.4) and (4.5) respectively.

Proof: Put $f(t)$ from (2.5) into (1.1), (3.6) and $\phi_{s}(t)$ from (3.9) into (3.7), together with considering mean (1.4), we get the followings respectively. 


$$
\begin{aligned}
& S_{f}(P, Q)=\sum_{i=1}^{n}\left(\frac{p_{i}+3 q_{i}}{4}\right) \log \left[\frac{p_{i}+3 q_{i}}{2\left(p_{i}+q_{i}\right)}\right]=M^{*}(P, Q) . \\
& B_{f}(\alpha, \beta)=\frac{(\beta-1) f(\alpha)+(1-\alpha) f(\beta)}{\beta-\alpha} \\
& =\frac{(\beta-1)(1+\alpha) \log \left(\frac{1+\alpha}{2 \alpha}\right)+(1-\alpha)(\beta+1) \log \left(\frac{1+\beta}{2 \beta}\right)}{2(\beta-\alpha)} . \\
& \left.B_{\phi_{s}}(\alpha, \beta)=\frac{(\beta-1) \phi_{s}(\alpha)+(1-\alpha) \phi_{s}(\beta)}{\beta-\alpha}\right] \\
& =[s(s-1)]^{-1}\left[\frac{(\beta-1)\left(\alpha^{s}-1\right)+(1-\alpha)\left(\beta^{s}-1\right)}{\beta-\alpha}\right] \\
& =[s(s-1)]^{-1}\left[\frac{\left(\beta^{s}-\alpha^{s}\right)}{\beta-\alpha}-\frac{\alpha \beta\left(\beta^{s-1}-\alpha^{s-1}\right)}{\beta-\alpha}-\frac{\beta-\alpha}{\beta-\alpha}\right] \\
& =[s(s-1)]^{-1}\left[s L_{s-1}(\alpha, \beta)-(s-1) \alpha \beta L_{s-2}(\alpha, \beta)-1\right], s \in R-\{0,1\} .
\end{aligned}
$$

Now let us consider the function $g(t)=t^{2-s} f^{\prime \prime}(t)=\frac{1}{2 t^{s}(t+1)}$, where $f^{\prime \prime}(t)$ is given by (2.6), and

$$
g^{\prime}(t)=-\left[\frac{(s+1) t+s}{2 t^{s+1}(t+1)^{2}}\right]=\left\{\begin{array}{l}
<0, s \geq 0 \\
>0, s \leq-1
\end{array}\right.
$$

So $g(t)$ is monotonically decreasing for $s \geq 0$ and monotonically increasing for $s \leq-1$.

Therefore, we have

$$
m=\inf _{t \in(\alpha, \beta)} g(t)=\left\{\begin{array}{l}
g(\beta)=\frac{1}{2 \beta^{s}(\beta+1)}, s \geq 0 \\
g(\alpha)=\frac{1}{2 \alpha^{s}(\alpha+1)}, s \leq-1
\end{array} .\right.
$$


$M=\sup _{t \in(\alpha, \beta)} g(t)=\left\{\begin{array}{l}g(\alpha)=\frac{1}{2 \alpha^{s}(\alpha+1)}, s \geq 0 \\ g(\beta)=\frac{1}{2 \beta^{s}(\beta+1)}, s \leq-1\end{array}\right.$.

The inequalities (4.1) and (4.2) are obtained by using (4.3), (4.4), (4.5), (4.7), and (4.8) in (3.5).

Now we consider some special cases of proposition 4 at $s=-1, s=0, s=1$, and at $s=2$ for getting bounds of the new divergence measure $M^{*}(P, Q)$, in terms of other divergences.

Result 4.1 Let $\Delta(P, Q)$ and $M^{*}(P, Q)$ be defined as in (1.3) and (2.7) respectively. For $P, Q \in \Gamma_{n}$, we have

$$
\begin{aligned}
& \frac{\alpha}{4(\alpha+1)}\left[2 \alpha \beta L_{-3}(\alpha, \beta)-L_{-2}(\alpha, \beta)-1-\frac{1}{2} \Delta(P, Q)\right] \leq B_{f}(\alpha, \beta)-M^{*}(P, Q) \\
& \leq \frac{\beta}{4(\beta+1)}\left[2 \alpha \beta L_{-3}(\alpha, \beta)-L_{-2}(\alpha, \beta)-1-\frac{1}{2} \Delta(P, Q)\right] .
\end{aligned}
$$

Proof: We evaluate $\Phi_{s}(Q, P)$ and $B_{\phi_{s}}(\alpha, \beta)$ at $s=-1$, i.e.,

$$
\begin{aligned}
& \Phi_{-1}(Q, P)=\frac{1}{2}\left[\sum_{i=1}^{n} q_{i}\left(\frac{p_{i}+q_{i}}{2 q_{i}}\right)^{-1}-1\right]=\frac{1}{2}\left[\sum_{i=1}^{n} \frac{2 q_{i}^{2}}{p_{i}+q_{i}}-\sum_{i=1}^{n} q_{i}\right]=\frac{1}{2}\left[\sum_{i=1}^{n} \frac{q_{i}^{2}-p_{i} q_{i}-p_{i} q_{i}+p_{i} q_{i}}{p_{i}+q_{i}}\right] \\
& =\frac{1}{2}\left[\sum_{i=1}^{n} \frac{q_{i}\left(p_{i}+q_{i}\right)}{\left(p_{i}+q_{i}\right)}-2 \sum_{i=1}^{n} \frac{p_{i} q_{i}}{p_{i}+q_{i}}\right]=\frac{1}{2}\left[1-2 \sum_{i=1}^{n} \frac{p_{i} q_{i}}{p_{i}+q_{i}}\right]=\frac{1}{2}\left[\sum_{i=1}^{n} \frac{p_{i}+q_{i}}{2}-2 \sum_{i=1}^{n} \frac{p_{i} q_{i}}{p_{i}+q_{i}}\right] \\
& =\frac{1}{4} \sum_{i=1}^{n} \frac{\left(p_{i}+q_{i}\right)^{2}-4 p_{i} q_{i}}{p_{i}+q_{i}}=\frac{1}{4} \sum_{i=1}^{n} \frac{\left(p_{i}-q_{i}\right)^{2}}{p_{i}+q_{i}}=\frac{1}{4} \Delta(P, Q) . \\
& B_{\phi_{-1}}(\alpha, \beta)=\frac{1}{2}\left[2 \alpha \beta L_{-3}(\alpha, \beta)-L_{-2}(\alpha, \beta)-1\right] .
\end{aligned}
$$

After putting (4.10) and (4.11) together with (4.4) in (4.2) at $s=-1$, we get the result (4.9) in terms of Triangular discrimination.

Result 4.2 Let $F(Q, P)$ and $M^{*}(P, Q)$ be defined as in (1.3) and (2.7) respectively. For $P, Q \in \Gamma_{n}$, we have

$$
\frac{1}{2(\beta+1)}\left[\log I\left(\frac{1}{\beta}, \frac{1}{\alpha}\right)-L_{-1}(\alpha, \beta)-1-F(Q, P)\right] \leq B_{f}(\alpha, \beta)-M^{*}(P, Q)
$$




$$
\leq \frac{1}{2(\alpha+1)}\left[\log I\left(\frac{1}{\beta}, \frac{1}{\alpha}\right)-L_{-1}(\alpha, \beta)-1-F(Q, P)\right] .
$$

Proof: We evaluate $\Phi_{s}(Q, P)$ and $B_{\phi_{s}}(\alpha, \beta)$ at $s \rightarrow 0$, i.e.,

$$
\begin{aligned}
& \Phi_{0}(Q, P)=\lim _{s \rightarrow 0} \Phi_{s}(Q, P)=\lim _{s \rightarrow 0}[s(s-1)]^{-1}\left[\sum_{i=1}^{n} q_{i}\left(\frac{p_{i}+q_{i}}{2 q_{i}}\right)^{s}-1\right] \\
& =\sum_{i=1}^{n} q_{i} \log \left(\frac{2 q_{i}}{p_{i}+q_{i}}\right)=F(Q, P) .
\end{aligned}
$$

$$
B_{\phi_{0}}(\alpha, \beta)=\lim _{s \rightarrow 0}\left[\frac{(\beta-1)\left(\alpha^{s}-1\right)+(1-\alpha)\left(\beta^{s}-1\right)}{s(s-1)(\beta-\alpha)}\right]=\frac{0}{0} .
$$

After applying D' Hospital Rule, we obtain

$$
\begin{aligned}
& B_{\phi_{0}}(\alpha, \beta)=\lim _{s \rightarrow 0}\left[\frac{(\beta-1)\left(\alpha^{s} \log \alpha\right)+(1-\alpha)\left(\beta^{s} \log \beta\right)}{(2 s-1)(\beta-\alpha)}\right]=\frac{\alpha \log \beta-\beta \log \alpha}{\beta-\alpha}-\frac{\log \beta-\log \alpha}{\beta-\alpha} \\
& =\log I\left(\frac{1}{\beta}, \frac{1}{\alpha}\right)-L_{-1}(\alpha, \beta)-1 .
\end{aligned}
$$

After putting (4.13) and (4.14) together with (4.4) in (4.1) at $s=0$, we get the result (4.12) in terms of Relative Jensen- Shannon divergence.

Result 4.3 Let $G(Q, P)$ and $M^{*}(P, Q)$ be defined as in (1.3) and (2.7) respectively. For $P, Q \in \Gamma_{n}$, we have

$$
\begin{aligned}
& \frac{1}{2 \beta(\beta+1)}\left[\log I(\alpha, \beta)-\alpha \beta L_{-1}(\alpha, \beta)-1-G(Q, P)\right] \leq B_{f}(\alpha, \beta)-M^{*}(P, Q) \\
& \leq \frac{1}{2 \alpha(\alpha+1)}\left[\log I(\alpha, \beta)-\alpha \beta L_{-1}(\alpha, \beta)-1-G(Q, P)\right] .
\end{aligned}
$$

Proof: We evaluate $\Phi_{s}(Q, P)$ and $B_{\phi_{s}}(\alpha, \beta)$ at $s \rightarrow 1$, i.e.,

$$
\Phi_{1}(Q, P)=\lim _{s \rightarrow 1} \Phi_{s}(Q, P)=\lim _{s \rightarrow 1}[s(s-1)]^{-1}\left[\sum_{i=1}^{n} q_{i}\left(\frac{p_{i}+q_{i}}{2 q_{i}}\right)^{s}-1\right]
$$




$$
\begin{aligned}
& =\sum_{i=1}^{n}\left(\frac{p_{i}+q_{i}}{2}\right) \log \left(\frac{p_{i}+q_{i}}{2 q_{i}}\right)=G(Q, P) . \\
& B_{\phi_{1}}(\alpha, \beta)=\lim _{s \rightarrow 1}\left[\frac{(\beta-1)\left(\alpha^{s}-1\right)+(1-\alpha)\left(\beta^{s}-1\right)}{s(s-1)(\beta-\alpha)}\right]=\frac{0}{0} .
\end{aligned}
$$

After applying D’ Hospital Rule, we obtain

$$
\begin{aligned}
& B_{\phi_{1}}(\alpha, \beta)=\lim _{s \rightarrow 1}\left[\frac{(\beta-1)\left(\alpha^{s} \log \alpha\right)+(1-\alpha)\left(\beta^{s} \log \beta\right)}{(2 s-1)(\beta-\alpha)}\right]=\frac{\beta \log \beta-\alpha \log \alpha}{\beta-\alpha}-\frac{\alpha \beta(\log \beta-\log \alpha)}{\beta-\alpha} \\
& =\log I(\alpha, \beta)-\alpha \beta L_{-1}(\alpha, \beta)-1 .
\end{aligned}
$$

After putting (4.16) and (4.17) together with (4.4) in (4.1) at $s=1$, we get the result (4.15) in terms of Relative Arithmetic- Geometric divergence.

Result 4.4 Let $\chi^{2}(P, Q)$ and $M^{*}(P, Q)$ be defined as in (1.3) and (2.7) respectively. For $P, Q \in \Gamma_{n}$, we have

$$
\begin{aligned}
& \frac{1}{4 \beta^{2}(\beta+1)}\left[2 L_{1}(\alpha, \beta)-(1+\alpha \beta)-\frac{1}{4} \chi^{2}(P, Q)\right] \leq B_{f}(\alpha, \beta)-M^{*}(P, Q) \\
& \leq \frac{1}{4 \alpha^{2}(\alpha+1)}\left[2 L_{1}(\alpha, \beta)-(1+\alpha \beta)-\frac{1}{4} \chi^{2}(P, Q)\right] .
\end{aligned}
$$

Proof: We evaluate $\Phi_{s}(Q, P)$ and $B_{\phi_{s}}(\alpha, \beta)$ at $s=2$, i.e.,

$$
\begin{aligned}
& \Phi_{2}(Q, P)=\frac{1}{2}\left[\sum_{i=1}^{n} q_{i}\left(\frac{p_{i}+q_{i}}{2 q_{i}}\right)^{2}-1\right]=\frac{1}{2}\left[\sum_{i=1}^{n} \frac{\left(p_{i}+q_{i}\right)^{2}}{4 q_{i}}-\sum_{i=1}^{n} p_{i}\right] \\
& =\frac{1}{8}\left[\sum_{i=1}^{n} \frac{\left(p_{i}-q_{i}\right)^{2}}{q_{i}}\right]=\frac{1}{8} \chi^{2}(P, Q) . \\
& B_{\phi_{2}}(\alpha, \beta)=\frac{1}{2}\left[2 L_{1}(\alpha, \beta)-(1+\alpha \beta)\right] .
\end{aligned}
$$

After putting (4.19) and (4.20) together with (4.4) in (4.1) at $s=2$, we get the result (4.18) in terms of Chi- Square divergence. 


\section{Bounds of the Relative J- divergence}

In this section, we obtain bounds of the Relative $J$ - divergence measure $J_{R}(P, Q)$ in terms of one parametric generalized divergence measure $\Phi_{s}(Q, P)$ for different values of $s$, by using new inequalities (3.5) and means (1.4) and (1.5).

Proposition 5 Let $\Phi_{s}(Q, P)$ and $J_{R}(P, Q)$ be defined as in (1.3) and (1.2) respectively. For $P, Q \in \Gamma_{n}$ , we have

a. For $s \rightarrow 0$ and $s<0$, we have

$$
\begin{aligned}
& \left(\alpha^{-s}+\alpha^{-s+1}\right)\left[B_{\phi_{s}}(\alpha, \beta)-\Phi_{s}(Q, P)\right] \leq B_{f}(\alpha, \beta)-\frac{1}{2} J_{R}(P, Q) \\
& \leq\left(\beta^{-s}+\beta^{-s+1}\right)\left[B_{\phi_{s}}(\alpha, \beta)-\Phi_{s}(Q, P)\right] .
\end{aligned}
$$

b. For $s \rightarrow 1$ and $s>1$, we have

$$
\begin{aligned}
& \left(\beta^{-s}+\beta^{-s+1}\right)\left[B_{\phi_{s}}(\alpha, \beta)-\Phi_{s}(Q, P)\right] \leq B_{f}(\alpha, \beta)-\frac{1}{2} J_{R}(P, Q) \\
& \leq\left(\alpha^{-s}+\alpha^{-s+1}\right)\left[B_{\phi_{s}}(\alpha, \beta)-\Phi_{s}(Q, P)\right]
\end{aligned}
$$

where $B_{f}(\alpha, \beta)$ is evaluated below by equation (5.6) and $B_{\phi_{s}}(\alpha, \beta)$ is given by (4.5).

Proof: Let $f:(0, \infty) \rightarrow R$ be a function defined as

$f(t)=(t-1) \log t$.

$f^{\prime}(t)=\frac{t-1}{t}+\log t, f(1)=0$ and

$$
f^{\prime \prime}(t)=\frac{t+1}{t^{2}} \text {. }
$$

Since $f^{\prime \prime}(t)>0 \forall t>0$ and $f(1)=0$, so $f(t)$ is convex and normalized function respectively.

Put $f(t)$ from (5.3) into (1.1) and (3.6), together with considering mean (1.4), we get the followings respectively.

$$
S_{f}(P, Q)=\frac{1}{2} \sum_{i=1}^{n}\left(p_{i}-q_{i}\right) \log \left(\frac{p_{i}+q_{i}}{2 q_{i}}\right)=\frac{1}{2} J_{R}(P, Q) .
$$




$$
\begin{aligned}
& B_{f}(\alpha, \beta)=\frac{(\beta-1) f(\alpha)+(1-\alpha) f(\beta)}{\beta-\alpha}=\frac{(\beta-1)(\alpha-1) \log \alpha+(1-\alpha)(\beta-1) \log \beta}{(\beta-\alpha)} \\
& =(\beta-1)(1-\alpha) \frac{(\log \beta-\log \alpha)}{(\beta-\alpha)}=(\beta-1)(1-\alpha) L_{-1}(\alpha, \beta) .
\end{aligned}
$$

Now let us consider the function $g(t)=t^{2-s} f^{\prime \prime}(t)=t^{2-s} \frac{(1+t)}{t^{2}}=t^{-s}+t^{-s+1}$, where $f^{\prime \prime}(t)$ is given by (5.4), and

$g^{\prime}(t)=-s t^{-s-1}+(1-s) t^{-s}=\left\{\begin{array}{c}<0, s \geq 1 \\ >0, s \leq 0\end{array}\right.$.

So $g(t)$ is monotonically decreasing for $s \geq 1$ and monotonically increasing for $s \leq 0$.

Therefore, we have

$$
\begin{gathered}
m=\inf _{t \in(\alpha, \beta)} g(t)=\left\{\begin{array}{ll}
g(\beta)=\left(\beta^{-s}+\beta^{-s+1}\right), & s \geq 1 \\
g(\alpha)=\left(\alpha^{-s}+\alpha^{-s+1}\right), & s \leq 0
\end{array} .\right. \\
M=\sup _{t \in(\alpha, \beta)} g(t)=\left\{\begin{array}{ll}
g(\alpha)=\left(\alpha^{-s}+\alpha^{-s+1}\right), & s \geq 1 \\
g(\beta)=\left(\beta^{-s}+\beta^{-s+1}\right), & s \leq 0
\end{array} .\right.
\end{gathered}
$$

The inequalities (5.1) and (5.2) are obtained by using (4.5), (5.5), (5.6), (5.8), and (5.9) in (3.5).

Now we consider some special cases of proposition 5 at $s=-1, s=0, s=1$, and at $s=2$ for getting bounds of the Relative $J$ - divergence measure $J_{R}(P, Q)$, in terms of other divergences.

Result 5.1 Let $\Delta(P, Q)$ and $J_{R}(P, Q)$ be defined as in (1.3) and (1.2) respectively. For $P, Q \in \Gamma_{n}$, we have

$$
\begin{aligned}
& \alpha(\alpha+1)\left[2 \alpha \beta L_{-3}(\alpha, \beta)-L_{-2}(\alpha, \beta)-1-\frac{1}{2} \Delta(P, Q)\right] \leq 2 B_{f}(\alpha, \beta)-J_{R}(P, Q) \\
& \leq \beta(\beta+1)\left[2 \alpha \beta L_{-3}(\alpha, \beta)-L_{-2}(\alpha, \beta)-1-\frac{1}{2} \Delta(P, Q)\right] .
\end{aligned}
$$

Proof: After putting (4.10) and (4.11) together with (5.6) in (5.1) at $s=-1$, we get the result (5.10) in terms of Triangular discrimination. We omit the detail.

Result 5.2 Let $F(Q, P)$ and $J_{R}(P, Q)$ be defined as in (1.3) and (1.2) respectively. For $P, Q \in \Gamma_{n}$, we have 


$$
\begin{aligned}
& 2(\alpha+1)\left[\log I\left(\frac{1}{\beta}, \frac{1}{\alpha}\right)-L_{-1}(\alpha, \beta)-1-F(Q, P)\right] \leq 2 B_{f}(\alpha, \beta)-J_{R}(P, Q) \\
& \leq 2(\beta+1)\left[\log I\left(\frac{1}{\beta}, \frac{1}{\alpha}\right)-L_{-1}(\alpha, \beta)-1-F(Q, P)\right] .
\end{aligned}
$$

Proof: After putting (4.13) and (4.14) together with (5.6) in (5.1) at $s=0$, we get the result (5.11) in terms of Relative Jensen- Shannon divergence. We omit the detail.

Result 5.3 Let $G(Q, P)$ and $J_{R}(P, Q)$ be defined as in (1.3) and (1.2) respectively. For $P, Q \in \Gamma_{n}$, we have

$$
\begin{aligned}
& \frac{2(\beta+1)}{\beta}\left[\log I(\alpha, \beta)-\alpha \beta L_{-1}(\alpha, \beta)-1-G(Q, P)\right] \leq 2 B_{f}(\alpha, \beta)-J_{R}(P, Q) \\
& \leq \frac{2(\alpha+1)}{\alpha}\left[\log I(\alpha, \beta)-\alpha \beta L_{-1}(\alpha, \beta)-1-G(Q, P)\right] .
\end{aligned}
$$

Proof: After putting (4.16) and (4.17) together with (5.6) in (5.2) at $s=1$, we get the result (5.12) in terms of Relative Arithmetic- Geometric divergence. We omit the detail.

Result 5.4 Let $\chi^{2}(P, Q)$ and $J_{R}(P, Q)$ be defined as in (1.3) and (1.2) respectively. For $P, Q \in \Gamma_{n}$, we have

$$
\begin{aligned}
& \frac{(\beta+1)}{\beta^{2}}\left[2 L_{1}(\alpha, \beta)-(1+\alpha \beta)-\frac{1}{4} \chi^{2}(P, Q)\right] \leq 2 B_{f}(\alpha, \beta)-J_{R}(P, Q) \\
& \leq \frac{(\alpha+1)}{\alpha^{2}}\left[2 L_{1}(\alpha, \beta)-(1+\alpha \beta)-\frac{1}{4} \chi^{2}(P, Q)\right] .
\end{aligned}
$$

Proof: After putting (4.19) and (4.20) together with (5.6) in (5.2) at $s=2$, we get the result (5.13) in terms of Chi-Square divergence. We omit the detail.

\section{Numerical verification of results obtained}

In this section, we give two examples for calculating the divergences $J_{R}(P, Q), \Delta(P, Q), M^{*}(P, Q)$ and verify the inequalities (Or bounds of $M^{*}(P, Q)$ and $J_{R}(P, Q)$ in terms of $\left.\Delta(P, Q)\right)(4.9)$ and (5.10).

Example 6.1 Let $P$ be the binomial probability distribution with parameters $(n=10, p=0.5)$ and $Q$ its approximated Poisson probability distribution with parameter $(\lambda=n p=5)$ for the random variable $X$, then we have 
Table 1: $(n=10, p=0.5, q=0.5)$

\begin{tabular}{|l|l|l|l|l|l|l|l|l|l|l|l|}
\hline$x_{i}$ & 0 & 1 & 2 & 3 & 4 & 5 & 6 & 7 & 8 & 9 & 10 \\
\hline$p\left(x_{i}\right)=p_{i} \approx$ & .000976 & .00976 & .043 & .117 & .205 & .246 & .205 & .117 & .043 & .00976 & .000976 \\
\hline$q\left(x_{i}\right)=q_{i} \approx$ & .00673 & .033 & .084 & .140 & .175 & .175 & .146 & .104 & .065 & .036 & .018 \\
\hline$\frac{p_{i}+q_{i} \approx}{2 q_{i}}$ & .573 & .648 & .757 & .918 & 1.086 & 1.203 & 1.202 & 1.063 & .831 & .636 & .527 \\
\hline
\end{tabular}

By using Table 1, we get the followings.

$\alpha(=0.527) \leq \frac{p_{i}+q_{i}}{2 q_{i}} \leq \beta(=1.203)$

$\Delta(P, Q)=\sum_{i=1}^{11} \frac{\left(p_{i}-q_{i}\right)^{2}}{p_{i}+q_{i}} \approx 0.0917$

$J_{R}(P, Q)=\sum_{i=1}^{11}\left(p_{i}-q_{i}\right) \log \left(\frac{p_{i}+q_{i}}{2 q_{i}}\right) \approx 0.0808$

$M^{*}(P, Q)=\sum_{i=1}^{11}\left(\frac{p_{i}+3 q_{i}}{4}\right) \log \left[\frac{p_{i}+3 q_{i}}{2\left(p_{i}+q_{i}\right)}\right] \approx 0.0076525$

Put the approximated numerical values from (6.1) to (6.4) in (4.9) and (5.10), we get

$$
9.110 \times 10^{-3} \leq 0.017059-0.007652\left(=M^{*}(P, Q)\right) \leq 0.014414
$$

and

$$
0.04248 \leq 0.169675-0.0404\left(=\frac{1}{2} J_{R}(P, Q)\right) \leq 0.13991
$$

respectively and hence verified the inequalities (4.9) and (5.10) for $p=0.5$.

Example 6.2 Let $P$ be the binomial probability distribution with parameters $(n=10, p=0.7)$ and $Q$ its approximated Poisson probability distribution with $\operatorname{parameter}(\lambda=n p=7)$ for the random variable $X$, then we have 
Table 2: $(n=10, p=0.7, q=0.3)$

\begin{tabular}{|l|l|l|l|l|l|l|l|l|l|l|l|}
\hline$x_{i}$ & 0 & 1 & 2 & 3 & 4 & 5 & 6 & 7 & 8 & 9 & 10 \\
\hline$p\left(x_{i}\right)=p_{i} \approx$ & .000059 & .000137 & .00144 & .009 & .036 & .102 & .20 & .266 & .233 & .121 & .0282 \\
\hline$q\left(x_{i}\right)=q_{i} \approx$ & .000911 & .00638 & .022 & .052 & .091 & .177 & .199 & .149 & .130 & .101 & .0709 \\
\hline$\frac{p_{i}+q_{i}}{2 q_{i}}$ & .503 & .510 & .532 & .586 & .697 & .788 & 1.002 & 1.392 & 1.396 & 1.099 & .698 \\
& & & & & & & & & & & \\
\hline
\end{tabular}

By using Table 2, we get the followings.

$\alpha(=0.503) \leq \frac{p_{i}+q_{i}}{2 q_{i}} \leq \beta(=1.396)$

$\Delta(P, Q)=\sum_{i=1}^{11} \frac{\left(p_{i}-q_{i}\right)^{2}}{p_{i}+q_{i}} \approx 0.1812$

$J_{R}(P, Q)=\sum_{i=1}^{11}\left(p_{i}-q_{i}\right) \log \left(\frac{p_{i}+q_{i}}{2 q_{i}}\right) \approx 0.1686$

$M^{*}(P, Q)=\sum_{i=1}^{11}\left(\frac{p_{i}+3 q_{i}}{4}\right) \log \left[\frac{p_{i}+3 q_{i}}{2\left(p_{i}+q_{i}\right)}\right] \approx 0.0115412$

Put the approximated numerical values from (6.5) to (6.8) in (4.9) and (5.10), we get

$$
0.01586 \leq 0.031810-0.0115412\left(=M^{*}(P, Q)\right) \leq 0.02762
$$

and

$$
0.03991 \leq 0.22497-0.0843\left(=\frac{1}{2} J_{R}(P, Q)\right) \leq 0.176588
$$

respectively and hence verified the inequalities (4.9) and (5.10) for $p=0.7$.

Similarly, we can verify the other inequalities (4.12), (4.15), (4.18), (5.11), (5.12), and (5.13). 


\section{Asymptotic approximation on new generalized f- divergence}

In this section, we introduce asymptotic approximation of $S_{f}(P, Q)$ in terms of well known divergence $\chi^{2}(P, Q)$ and get the approximate relations of $M^{*}(P, Q)$ and $J_{R}(P, Q)$ with $\chi^{2}(P, Q)$, respectively.

Theorem 7.1 If $f:(0, \infty) \rightarrow R$ is twice differentiable, convex, and normalized function, i.e., $f^{\prime \prime}(t)>0$ and $f(1)=0$ respectively, then we have

$S_{f}(P, Q) \approx \frac{f^{\prime \prime}(1)}{8} \chi^{2}(P, Q)$.

Equivalently

$$
\left|\frac{S_{f}(P, Q)}{\chi^{2}(P, Q)}-\frac{f^{\prime \prime}(1)}{8}\right|<\varepsilon \text { when }|P-Q|<\delta,
$$

where $\varepsilon, \delta \rightarrow 0$, i.e., $\varepsilon, \delta$ are very small and $S_{f}(P, Q), \chi^{2}(P, Q)$ are given by (1.1) and (1.3) respectively.

Proof: We know by Taylor's series expansion of function $f(t)$ at $t=1$, that

$f(t)=f(1)+(t-1) f^{\prime}(1)+\frac{(t-1)^{2}}{\lfloor 2} f^{\prime \prime}(1)+(t-1)^{2} g(t)$,

where $g(t)=\frac{(t-1)}{\lfloor 3} f^{\prime \prime \prime}(1)+\frac{(t-1)^{2}}{4} f^{\prime \prime \prime \prime}(1)+\ldots$ and we can see that $g(t) \rightarrow 0$ as $t \rightarrow 1, f(1)=0$ because $f(t)$ is normalized, therefore from (7.2) we get

$$
f(t) \approx(t-1) f^{\prime}(1)+\frac{(t-1)^{2}}{\lfloor 2} f^{\prime \prime}(1)
$$

Now Put $t=\frac{p_{i}+q_{i}}{2 q_{i}}$ in (7.3), multiply with $q_{i}$ and then sum over all $i=1,2, \ldots, n$, we get the desire result (7.1).

Remark: Particularly if we take $f(t)=\frac{(t+1)}{2} \log \left(\frac{t+1}{2 t}\right)$ and $(t-1) \log t$ in (7.1), we get $M^{*}(P, Q) \approx \frac{1}{32} \chi^{2}(P, Q), J_{R}(P, Q) \approx \frac{1}{2} \chi^{2}(P, Q)$ respectively, where $J_{R}(P, Q)$ and $M^{*}(P, Q)$ are given by (1.2) and (2.7) respectively. 


\section{Conclusion and discussion}

In this work, we introduced new information divergence measure, characterized, defined the properties and derived relations with other divergences. Asymptotic approximation of this new divergence and Relative J- divergence has been derived as well. Two new information inequalities on $S_{f}(P, Q)$ in terms of standard one parametric generalized divergence have been introduced and further bounds of the new divergence and Relative J- divergence have been obtained in terms of the other well known divergences in an interval $(\alpha, \beta), 0<\alpha \leq 1 \leq \beta<\infty$ with $\alpha \neq \beta$ as an application of new information inequalities. These bounds have been verified numerically by taking two discrete distributions: Binomial and Poisson.

We found in articles $[3, \mathbf{1 6}]$ that square root of some particular divergences is a metric space but not each because of violation of triangle inequality, so we strongly believe that divergence measures can be extended to other significant problems of functional analysis and its applications, such investigations are actually in progress because this is also an area worth being investigated. Such types of divergences are also very useful to find the mutual information $[12,13,17]$, i.e., how much information is conveyed of one random variable about other and utility of an event $[4, \mathbf{3 0}]$, i.e., an event is how much useful compare to other event.

We hope that this work will motivate the reader to consider the extensions of divergence measures in information theory, other problems of functional analysis and fuzzy mathematics.

\section{References}

[1] Bajaj R. K, Hooda D.S., "Generalized measures of fuzzy directed divergence, total ambiguity and information improvement", Journal of Applied Mathematics, Statistics and Informatics, vol. 6 (2010), no. 2, pp: 31- 44.

[2] Bassat M.B., "f- Entropies, probability of error and feature selection", Inform. Control, vol. 39 (1978), pp: 227-242.

[3] Bhatia P.K, Singh S., “On a new Csiszar's f-divergence measure”, Cybernetics and Information Technologies, vol. 13 (2013), no. 2, pp: 43- 56.

[4] Bhullar J.S., Vinocha O.P., Gupta M., "Generalized measure for two utility distributions", Proceedings of the World Congress on Engineering, vol. 3 (2010), June 30- July 2.

[5] Bregman L.M., "The relaxation method to find the common point of convex sets and its applications to the solution of problems in convex programming," USSR Comput. Math. Phys., vol. 7 (1967), pp: $200-217$.

[6] Burbea J., Rao C.R, "On the convexity of some divergence measures based on entropy functions", IEEE Trans. on Inform. Theory, IT-28 (1982), pp: 489-495.

[7] Chen H.C., "Statistical pattern recognition", Hoyderc Book Co., Rocelle Park, New York, (1973).

[8] Chow C.K., Lin C.N., "Approximating discrete probability distributions with dependence trees", IEEE Trans. Inform. Theory, vol. 14 (1968), no 3, pp: 462-467.

[9] Csiszar I., "Information measures: A Critical survey", in Trans. - In: Seventh Prague Conf. on Information Theory, Academia, Prague, (1974), pp: 73-86.

[10] Csiszar I., "Information type measures of differences of probability distribution and indirect observations", Studia Math. Hungarica, vol. 2 (1967), pp: 299- 318.

[11] Dacunha- Castelle D., "Ecole d'Ete de Probabilites de", Saint-Flour VII-1977, Berlin, Heidelberg, New York: Springer, (1978). 
[12] Dragomir S.S., Gluscevic V., Pearce C.E.M., “Approximation for the Csiszar's $f$ - divergence via midpoint inequalities", in Inequality Theory and Applications - Y.J. Cho, J.K. Kim, and S.S. Dragomir (Eds.), Nova Science Publishers, Inc., Huntington, New York, Vol. 1 (2001), pp: 139154.

[13] Dragomir S.S., Scholz M.L., Sunde J., "Some upper bounds for relative entropy and applications", Computers and Mathematics with Applications, vol. 39 (2000), pp: 91- 100.

[14] Gokhale D.V., Kullback S., "Information in contingency Tables", New York, Marcel Dekker, (1978).

[15] Hooda D.S., “On generalized measures of fuzzy entropy”, Mathematica Slovaca, vol. 54 (2004), no. 3, pp: 315- 325 .

[16] Jain K.C., Chhabra P., "Series of new information divergences, properties and corresponding series of metric spaces", International Journal of Innovative Research in Science, Engineering and Technology, vol. 3, no.5 (2014), pp: 12124- 12132.

[17] Jain K.C., Chhabra P., "New series of information divergence measures and their properties", Communicated.

[18] Jain K.C., Saraswat R.N., "Some new information inequalities and its applications in information theory", International Journal of Mathematics Research, vol. 4, no.3 (2012), pp: 295- 307.

[19] Jha P., Mishra V. K., "Some new trigonometric, hyperbolic and exponential measures of fuzzy entropy and fuzzy directed divergence", International Journal of Scientific and Engineering Research, vol. 3 (2012), no. 4, pp: 1- 5.

[20] Jones L., Byrne C., "General entropy criteria for inverse problems with applications to data compression, pattern classification and cluster analysis", IEEE Trans. Inform. Theory, vol. 36, (1990), pp: 23- 30.

[21] Kadota T.T., Shepp L.A., "On the best finite set of linear observables for discriminating two Gaussian signals", IEEE Trans. Inform. Theory, vol. 13, (1967), pp: 288-294.

[22] Kailath T, "The divergence and Bhattacharyya distance measures in signal selection", IEEE Trans. Comm. Technology, vol. COM-15, (1967), pp: 52- 60.

[23] Kazakos D., Cotsidas T, "A decision theory approach to the approximation of discrete probability densities", IEEE Trans. Perform. Anal. Machine Intell, vol. 1, 1980, pp: 61- 67.

[24] Nielsen F., Boltz S., "The Burbea-Rao and Bhattacharyya centroids", Apr. (2010), Arxiv.

[25] Pearson K., "On the Criterion that a given system of deviations from the probable in the case of correlated system of variables is such that it can be reasonable supposed to have arisen from random sampling", Phil. Mag., 50 (1900), pp: 157-172.

[26] Pielou E.C, "Ecological diversity”, New York, Wiley, (1975).

[27] Renyi A., "On measures of entropy and information", Proc. 4th Berkeley Symposium on Math. Statist. and Prob., 1 (1961), pp: 547-561.

[28] Santos-Rodriguez R., Garcia-Garcia D., Cid-Sueiro J., "Cost-sensitive classification based on Bregman divergences for medical diagnosis", In M.A. Wani, editor, Proceedings of the $8^{\text {th }}$ International Conference on Machine Learning and Applications (ICMLA'09), Miami Beach, Fl., USA, December 13-15, (2009), pp: 551- 556, 2009.

[29] Sibson R., "Information radius", Z. Wahrs. Undverw. Geb., (14) (1969), pp: 149-160.

[30] Taneja H.C., Tuteja R.K., "Characterization of a quantitative- qualitative measure of inaccuracy", Kybernetika, vol. 22 (1986), no. 5, pp: 393- 402.

[31] Taneja I.J., "New developments in generalized information measures", Chapter in: Advances in Imaging and Electron Physics, Ed. P.W. Hawkes, 91(1995), pp: 37-135.

[32] Taneja I.J., Kumar P., "Generalized non-symmetric divergence measures and inequalities", The Natural Science and Engineering Research Council's Discovery grant to Pranesh Kumar (2000).

[33] Taskar B., Lacoste-Julien S., Jordan M.I., "Structured prediction, dual extra gradient and Bregman projections", Journal of Machine Learning Research, 7 (2006), pp: 1627- 1653.

[34] Theil H., "Statistical decomposition analysis", Amsterdam, North-Holland, (1972). 
[35] Theil H., "Economics and information theory", Amsterdam, North-Holland, (1967).

[36] Vemuri B., Liu M., Amari S., Nielsen F., "Total Bregman divergence and its applications to DTI analysis", IEEE Transactions on Medical Imaging, (2010). 